Selbstmonitoring von Gewicht und Ernährung, Bewegung, Zielvereinbarungen, Ernährungsberatung, Bewegungsempfehlungen, Problemlösungen und Rückfallpropyhlaxe den Therapieerfolg erhöhen.

$\mathrm{Zu}$ ähnlichen Ergebnissen kam eine zweijährige Studie in Praxen der Primärversorgung in den USA (Wadden TA et al. NEJM 2011;365:1969-1979). Ein Standardprogramm (5-7 Minuten Beratung alle drei Monate) verminderte das Gewicht um 1,7 kg, ein Coaching durch einen Lifestyle-Berater (10-15 Minuten alle drei Monate) um 2,9 kg.
Diese beiden Untersuchungen geben Anlass zum Umdenken:

- Schon kurze Konsultationen in der Praxis des niedergelassenen Arztes reduzieren das Gewicht.

- Die parallele Betreuung in kommerziellen Programmen verdoppelt den Erfolg. Ärzte sollten ihre Patienten zur Teilnahme ermuntern.

In den nächsten Jahren wird sich zeigen, welche Bedeutung neben der Betreuung in Praxen von niedergelassenen Ärzten gemeindebasierte Programme, Programme in
KITAs und Schulen oder EDV-gestützte Programme (Telefonkontakt, Internet, Telemonitoring) haben.

Prof. Dr. med. Alfred Wirth, Bad Rothenfelde II

In Jebb SA et al. Primary care referral to a commercial provider for weight loss treatment versus standard care: a randomised controlled trial. Lancet 2011;378:1485-1492

\title{
Gesundheitsrisiko Luftverschmutzung
}

\section{Mehr Schlaganfälle bei hoher Feinstaubbelastung}

\section{Luftverschmutzung belastet nicht nur die Atemwege. Ein Anstieg der Feinstaubbelastung in der Luft führte in einer amerikanischen Studie bereits nach wenigen Stunden zu einem erhöhten Schlaganfallrisiko.}

In die retrospektive Beobachtungsstudie wurden 1705 Patienten aufgenommen, die zwischen 1999 und 2008 wegen eines akuten ischämischen Schlaganfalls in einem Krankenhaus der Harvard Medical School in Boston stationär behandelt wurden. Während des gleichen Zeitraums wurde die Konzentration von Feinstaub $<2,5 \mu \mathrm{m}$ (PM 2,5) und anderen Substanzen in der Umgebungsluft an der nahegelegenen Messstation der Harvard University kontinuierlich gemessen. Alle Patienten lebten im Umkreis von $40 \mathrm{~km}$ um diese Messstation. Zur Untersuchung des Einflusses der Feinstaubbelastung auf das Schlaganfallrisiko führten die Wissenschaftler eine Fallkontrollstudie mit Case-Crossover-Design durch.

Es zeigte sich, dass das Schlaganfallrisiko während einer 24-Stunden-Periode mit moderater Feinstaubkonzentration (15-40 $\mu \mathrm{g} /$ $\mathrm{m}^{3}$ ) um $34 \%$ erhöht war im Vergleich zu 24-Stunden-Perioden mit geringer Feinstaubkonzentration $\left(\leq 15 \mu \mathrm{g} / \mathrm{m}^{3}\right)$.

Über den gesamten Bereich der gemessenen Feinstaubkonzentrationen hinweg fand sich ein Anstieg des Schlaganfallrisikos um $11 \%$ pro Zunahme der Konzentration um
$6,4 \mu \mathrm{g} / \mathrm{m}^{3}$. Der Anstieg des Schlaganfallrisikos war dabei am größten innerhalb von 12-24 Stunden nach einer erhöhten Feinstaubbelastung.

Ähnliche Zusammenhänge mit dem Schlaganfallrisiko fanden sich auch für höhere Konzentrationen von Ruß und Stickstoffdioxid, nicht aber für andere Luftschadstoffe. Andere Luftparameter (Temperatur, Luftdruck, Ozon) und vaskuläre Risikofaktoren hatten keinen messbaren Einfluss auf den Zusammenhang zwischen Feinstaubbelastung und Schlaganfallrisiko.

II Kommentar: Es ist mittlerweile gut belegt, dass eine erhöhte Feinstaubbelastung der Umgebungsluft zahlreiche Gesundheitsschäden auslöst und neben Atemwegserkrankungen auch das Risiko für Herzinfarkt, arterielle Verschlusskrankheit, Mortalität und Krankenhausbehandlung erhöht. Die vorliegende Studie belegt, dass bei höheren Feinstaubbelastungen auch das Risiko eines Schlaganfalls ansteigt. Ursächlich können dabei inflammatorische, hämostatische, endotheliale und vegetative Prozesse eine Rolle spielen.

Die Tatsache, dass neben der Feinstaubkonzentration auch erhöhte Konzentrationen von Ruß und Stickstoffdioxid das Schlaganfallrisiko erhöhten, deutet nach Einschätzung der Autoren vor allem auf die Bedeutung des Straßenverkehrs als Hauptquelle der Schädigung hin. Auch wenn die indivi- duelle Risikoerhöhung für den Einzelnen dabei gering sein mag, ergibt sich aufgrund der sehr weiten Verbreitung erhöhter Feinstaubkonzentrationen und anderer Luftbelastungen durch Straßenverkehr auf Bevölkerungsebene ein hohes populationsattributables Risiko. Den anhaltenden Bemühungen der nationalen und internationalen Umweltbehörden zur Absenkung der Feinstaubbelastung in der Umgebungsluft kommt somit eine große Bedeutung für den Gesundheitsschutz der Bevölkerung zu.

Dr. med. Markus Busch, Robert Koch-Institut Berlin II

II Wellenius GA et al. Ambient air pollution and the risk of acute ischemic stroke. Arch Intern Med 2012;172:229-234

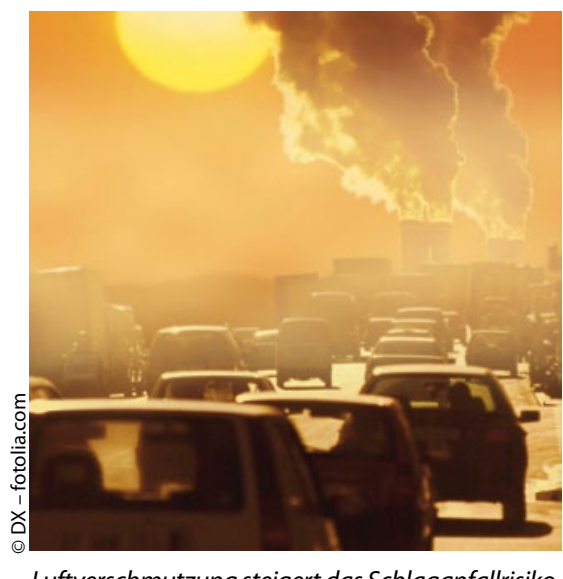

